\title{
Bridging therapy to chronic anticoagulant treatment in patients undergoing cardiac surgery: comparison between low-molecular-weight heparin and subcutaneous unfractionated heparin. A pilot study
}

\author{
Carlo Rostagno $^{1 *}$ and Pier Luigi Stefàno ${ }^{2}$ \\ ${ }^{1}$ Dipartimento Medicina Sperimentale e Clinica, Università di Firenze, Italy \\ ${ }^{2}$ SOD Cardiochirurgia , AOU Careggi Firenze, Italy
}

\begin{abstract}
Background: At present, no clear evidence has been provided regarding the superiority of various heparin regimens as bridging treatment after heart surgery in patients who need chronic anticoagulation.

Objectives: Aim of present pilot investigation was to prospectively compare the safety of weight adjusted enoxaparin and subcutaneous unfractionated heparin in patients undergoing valvular heart surgery.

Methods: Patients undergoing heart valve surgery were randomized to weight adjusted enoxaparin (group I) or comparable weight adjusted subcutaneous unfractionated heparin (group II) started within 12 hours after ICU admission. As control group in the same period patients undergoing isolated CABG were randomized to either treatment (group III and IV). Hemorrhagic and thromboembolic complications, the need for blood transfusions, total drainage fluid, haemoglobin values and presence and degree of pericardial effusion at discharge were evaluated.

Results: 184 patients were included in the study 69 in group I and 115 in group II. Twenty and respectively 49 patients were enrolled in the control groups. Average fluid drainage after 24 hours was similar with the two regimens both in study and control group. A trend towards the need of blood unit transfusion in ICU after starting heparin therapy was higher in patients treated with. s.c. unfractionated heparin vs enoxaparin ( 40 vs. $30 \% \mathrm{p}<0.05)$. No thromboembolic events were recorded. Hemorrhagic pericardial effusion requiring surgical drainage was more frequent with unfractionated heparin ( 7 vs. 1) however all patients went to our observation after treatment withdrawal, usually between 10 and 17 days after surgery and all but one had anticoagulant over dosage (average INR 4.53). In control groups only one patient needed pericardiocentesis.
\end{abstract}

Conclusion: In patients who need prolonged oral anticoagulation hospital bridging treatment with weight adjusted enoxaparin seems as safe as weight adjusted s.c unfractionated heparin with a lower rate of blood transfusion and a lower rate of pericardial effusion and need for pericardial drainage.

\section{Backgound}

After heart valve surgery requiring prolonged anticoagulation no clear evidence has still been provided regarding the safer and more effective bridging anticoagulant treatment to be started on first postoperative day and prosecuted until full effectiveness of vitamin $\mathrm{K}$ antagonist treatment [1] . The risk of bleeding early after surgery must be faced to the risk of thromboembolic events in the subsequent days. Unfractionated heparin (UFH) has for a long term been the standard bridging anticoagulant drug [2], however low-molecular-weight heparin (LMWH) may be as safe and effective without increase of bleeding risk [3]. LMWH has more predictable and rapidly reached anticoagulation due to its better Few studies have analyzed LMWH as a bridging anticoagulant after valve replacement or repair [4-6].

The purpose of present pilot investigation was to prospectively compare the safety of weight adjusted enoxaparin and subcutaneous unfractionated heparin in patients undergoing valvular heart surgery.

\section{Method}

From January to June 2012, 214 patients underwent heart valve surgery at our institution. Exclusion criteria from the study were a recent neurologic event, pre- or post-operative severe renal insufficiency (serum creatinine $>200 \mu \mathrm{mol} / \mathrm{L}$ ), dialysis, aortic dissection, critical perioperative state, intraaortic balloon counter pulsation or duration of intubation of more than 48 hours. 184 patients were therefore included in the investigation.

\section{Study design}

After surgery, they were randomized to weight adjusted enoxaparin (January-March) or comparable weight adjusted subcutaneous

Correspondence to: Dr. Carlo Rostagno, Largo Brambilla 3, 50134 Firenze, Italy, Tel: +390557945845; Fax: +390557948547; E-mail : carlo.rostagno@unifi.it

Key words: anticoagulants, valves, stroke, heart valve surgery, haemorrhage

Received: April 15, 2017; Accepted: May 23, 2017; Published: May 26, 2017 
Rostagno C (2017) Bridging therapy to chronic anticoagulant treatment in patients undergoing cardiac surgery: comparison between low-molecular-weight heparin and subcutaneous unfractionated heparin. A pilot study

unfractionated heparin (April -June) started within 12 hours after ICU admission. As control group in the same period patients undergoing isolated CABG were randomized to either treatment (group III and IV).

Postoperative anticoagulation protocol was started on postoperative day 1 , with 1 weight adjusted subcutaneous enoxaparin or sc UFH injection per day (Table 1). Oral anticoagulation (warfarin) was started as soon as drainage tubes were removed. On postoperative day 2, subcutaneous enoxaparin and UFH were administered at 12hour intervals. LMWH and sc UFH was given until the International Normalized Ratio (INR) was within the target range for 2 consecutive days (INR 2 to 3 after aortic valve replacement or 2.5 to 3.5 after mitral or tricuspid valve replacement). Antiplatelet therapy was only prescribed in case of concomitant peripheral vascular disease or coronary artery disease. Echocardiography was systematically performed prior to discharge, usually on post-operative day. Hemorrhagic and thromboembolic complications, the need for blood transfusions, total drainage fluid, hemoglobin values and presence and degree of pericardial effusion at discharge other than the need for pericardial drainage were evaluated. Written informed consent was obtained at hospital admission. The study was approved by Ethical Committee of the Azienda Ospedaliera Universitaria Careggi, Firenze.

\section{End-points}

The primary end points of this study were the occurrence of a thromboembolic (efficacy end point) or major bleeding (safety end point) event during the 4-week follow-up. Thromboembolic events were defined as a transient or permanent stroke, peripheral embolism or valve thrombosis. Major bleeding events were defined as any bleeding requiring transfusion, surgical operation, or prolonged hospitalization or rehospitalization

\section{Statistical analysis}

The data are presented as the mean \pm standard deviation (SD). Differences between the categorical variables were tested using Fisher's exact test. Differences between continuous variables were tested using the Student's t-test. A P-value of less than 0.05 was considered to be statistically significant.

Perioperative variations in the hemoglobin level, haematocrit in drainage fluid, fluid drainage at 24 hours, mean unit of blood transfused were analyzed using a one-way repeated ANOVA test. The statistical computations were performed using SPSS (SPSS ${ }^{\bullet}$ version 17.0, Chicago, IL).

\section{Results}

Baseline demographic, clinical and operative characteristics are reported in Table 2 and 3

During the 4 weeks after surgery, no patients had thromboembolic event thus suggesting that both enoxaparin and sc UH may be efficient as bridge therapy in patients with indication to long term oral anticoagulation. We found a trend towards a higher fluid drainage after 24 hours in patients treated with unfractionated heparin in comparison to LMWH. A similar trend was found also in control group e.g patients undergoing isolated myocardial revascularization. The need for blood transfusion in ICU and during the whole hospital stay was not significantly different in the two groups although UFH showed a trend for a higher need (Table 4). There was no difference in haemoglobin levels at discharge between patients treated with UFH and LMWH. At hospital discharge, there was no significant difference in the incidence of mild to moderate pericardial effusion in patient treated with dose
Table 1. Heparin dosage

\begin{tabular}{|c|c|c|}
\hline Weight $(\mathrm{kg})$ & Enoxaparin dose & Sc UFH dose \\
\hline$<50$ & 2000 & 5000 \\
\hline $50-69$ & 3000 & 7500 \\
\hline $70-89$ & 4000 & 12500 \\
\hline$>90$ & 6000 & 12500 \\
\hline
\end{tabular}

Table 2. Clinical characteristics of patients

\begin{tabular}{|c|c|c|}
\hline Characteristics & LMWH & UH \\
\hline $\mathrm{N}$ & 69 & 115 \\
\hline Age (years \pm SD) & $69.4( \pm 11.3)$ & $68.1( \pm 11.8)$ \\
\hline Hypertension (\%) & $49(59)$ & $70(56.43)$ \\
\hline FA history (\%) & $18(26)$ & $39(33)$ \\
\hline LA diameter (mm) & $44.65 \pm 6.8)$ & $43.96 \pm 7.1$ \\
\hline LVEF (\%) & $51.3 \pm 10.5$ & $51.9 \pm 12.2$ \\
\hline$\beta-$ Blockers (\%) & $55(79)$ & $83(72)$ \\
\hline $\begin{array}{c}\text { Ace- inhibitors or AT }- \\
\text { blocker (\%) }\end{array}$ & $61(88)$ & $103(89)$ \\
\hline
\end{tabular}

Table 3. Type of surgery

\begin{tabular}{|c|c|c|c|c|}
\hline & \multicolumn{2}{|c|}{ ENOXAPARIN } & \multicolumn{2}{c|}{ sc UFH } \\
\cline { 2 - 5 } & No CABG & CABG & NO-CABG & CABG \\
\hline $\begin{array}{c}\text { AORTIC VALVE } \\
\text { REPLACEMENT }\end{array}$ & 13 & 10 & 24 & 15 \\
\hline $\begin{array}{c}\text { AORTIC ROOT SURG. } \\
\text { (main Bentall) }\end{array}$ & 11 & & 18 & \\
\hline $\begin{array}{c}\text { MITRAL VALVE SURGERY } \\
\text { (75\% MV repair) }\end{array}$ & 14 & 5 & 24 & 9 \\
\hline $\begin{array}{c}\text { MITRAL AND AORTIC } \\
\text { REPLACEMENT }\end{array}$ & 7 & 5 & 11 & 10 \\
\hline OTHER & 3 & & 4 & \\
\hline
\end{tabular}

Table 4. Haematocrit $\%$ in drainage fluid, total fluid drainage at 24 hours, mean blood. Unit transfused and mean haemoglobin at discharge in the 4 groups under investigation.

\begin{tabular}{|c|c|c|c|c|}
\hline & $\begin{array}{c}\text { GROUP I } \\
\text { LMWH }\end{array}$ & $\begin{array}{c}\text { GROUP II SC } \\
\text { UHEP }\end{array}$ & $\begin{array}{c}\text { GROUP III } \\
\text { LMWH CABG }\end{array}$ & $\begin{array}{c}\text { GROUP IV } \\
\text { UHEP CABG }\end{array}$ \\
\hline $\begin{array}{c}\text { Haematocrit \% } \\
\text { in drainage fluid } \\
8 \text { hours PO }\end{array}$ & $12.7 \pm 6.5$ & $13.9 \pm 13.4$ & $15.3 \pm 7.3$ & $12.7 \pm 7.8$ \\
\hline $\begin{array}{c}\text { Haematocrit \% } \\
\text { in drainage fluid } \\
\text { 16 hours PO }\end{array}$ & $8.3 \pm 5.1$ & $8.4 \pm 6.3$ & $8.2 \pm 5.1$ & $7.5 \pm 4.9$ \\
\hline $\begin{array}{c}\text { Fluid drainage at } \\
\text { 24 hours (ml) }\end{array}$ & $418.4 \pm 210$ & $469 \pm 299$ & $409 \pm 230$ & $510 \pm 291$ \\
\hline $\begin{array}{c}\text { Mean Blood } \\
\text { Unit transfused }\end{array}$ & $1.2 \pm 1.3$ & $1.9 \pm 2.4$ & $0.7 \pm 0.9$ & $0.9 \pm 1.2$ \\
\hline $\begin{array}{c}\text { Haemoglobin at } \\
\text { discharge }\end{array}$ & $10.2 \pm 1.23$ & $10.3 \pm 2.2$ & $10.4 \pm 1.4$ & $10.3 \pm 1.7$ \\
\hline
\end{tabular}

adjusted UH in comparison to patients in LMWH (18 \% vs. 19\% respectively). Hemorrhagic pericardial effusion requiring drainage however was significantly more frequent in patients treated with unfractionated heparin (6.1\%) than with enoxaparin (1.8\%) (Table 5). Pericardial drainage was performed in 3 group II (UFH) patients before hospital discharge, while the other procedures were performed largely after treatment withdrawal, between 10 and 17 days after surgery. All but one patients had INR was above therapeutic level (average value 4.53). No heparin-induced thrombocytopenia was diagnosed during the follow-up period.

\section{Discussion}

The bridging regimen after heart surgery needing long term oral anticoagulation is still not clearly defined [7-9]. ESC guidelines [10] suggest that oral anticoagulation should be started during the first 
Rostagno C (2017) Bridging therapy to chronic anticoagulant treatment in patients undergoing cardiac surgery: comparison between low-molecular-weight heparin and subcutaneous unfractionated heparin. A pilot study

Table 5. Pericardial complications in the 4 groups

\begin{tabular}{|c|c|c|c|c|}
\hline & $\begin{array}{c}\text { GROUP } \\
\text { I LMWH } \\
\mathbf{n = 6 9}\end{array}$ & $\begin{array}{c}\text { GROUP II } \\
\text { SC UHEP } \\
\mathbf{n = 1 1 5}\end{array}$ & $\begin{array}{c}\text { GROUP } \\
\text { III LMWH } \\
\text { CABG n=20 }\end{array}$ & $\begin{array}{c}\text { GROUP IV } \\
\text { UHEP } \\
\text { CABG n=49 }\end{array}$ \\
\hline No pericardial effusion & 53 & 88 & 17 & 46 \\
\hline $\begin{array}{c}\text { Mild pericardial } \\
\text { Effusion }\end{array}$ & 9 & 12 & 2 & 2 \\
\hline Moderate pericardial effusion & 6 & 8 & - & 1 \\
\hline $\begin{array}{c}\text { Effusion requring pericardial } \\
\text { drainage }\end{array}$ & 1 & 7 & 1 & - \\
\hline
\end{tabular}

postoperative days. Intravenous unfractionated heparin (UFH), monitored to an activated partial thromboplastin time (aPTT) of 1.52.0 times control value, enables rapid anticoagulation to be obtained before the INR rises. Low molecular weight heparin (LMWH) seems to offer effective and stable anticoagulation and has been used in small observational series but there are few randomized trials supporting this practice [4]. In the case of LMWH administration anti-Xa monitoring is recommended by guidelines. AHA/ACC guidelines [11] indicates that bridging anticoagulation is typically started once postoperative bleeding is no longer an issue. Some centers use subcutaneous lowmolecular-weight heparin (LMWH) or unfractionated heparin (UFH), whereas other centers continue to prefer intravenous UFH.

In our prospective randomized study, we compared weight adjusted enoxaparin with weight adjusted subcutaneous unfractionated heparin. Result from this investigation suggest that that the administration of enoxaparin as a bridging therapy immediately after heart valve surgery requiring long term anticoagulation is effective, and safe as sc UFH Major bleeding and in particular haemorrhagic pericardial effusion with the need for pericardial drainage have been recorded more frequently in the subgroup of patients treated with UH. This finding however may be considered with caution since $>50 \%$ of haemo -pericardium requiring pericardial drainage occurred late, after hospital discharge to rehabilitation centres, and all, except one, had INR largely above therapeutic range. In our anticoagulation protocol, only prophylactic enoxaparin and UFH were given on postoperative day 1 to reduce the risk of bleeding. Indeed, significant haemostasis alterations were reported during the first 24 hours following cardiopulmonary bypass surgery Vitamin $\mathrm{K}$ agonist therapy generally began on the first postoperative day to ensure a shorter LMWH anticoagulation bridging duration. This may explain the absence of heparin- induced thrombocytopenia in our study.

Our findings, in terms of thromboembolic and major bleeding event rates, are consistent with those previously reported based on the use of UH or LMWH anticoagulation therapy after heart surgery. Subcutaneous enoxaparin was used as bridging anticoagulant treatment in a prospective registry of patients who underwent cardiac surgery between December 2003 and June 2004 and required long-term anticoagulation [4]. The mean (SD) number of days between surgery and the first dose of anticoagulant was 2.01 for acenocumarol and 1 for enoxaparin. The mean (SD) daily dose of enoxaparin was $1.1(0.27) \mathrm{mg} /$ kg. Six thromboembolic events (4.3\%; 95\% CI 1.6 to 9.1) occurred, but only four of them were plausibly related to enoxaparin $(2.9 \%$; $95 \%$ CI 0.8 to 7.1). Six major hemorrhagic events ( $4.3 \%$; $95 \%$ CI 1.6 to 9.1$)$ occurred, but only three were plausibly related to enoxaparin $(2.1 \%$; $95 \%$ CI 0.4 to 6.1). Bucci et al. [6] in a retrospective study compared dalteparin $(\mathrm{n}=100)$ as postoperative anticoagulant with UFH $(\mathrm{n}=103)$. Overall, there were for fewer thrombotic events in the LMWH-treated group ( $4 \%$ vs. $11 \%, \mathrm{p}=0.11)$. There was a higher rate of bleeding events in the UFH-treated group $(10 \%$ vs. $3 \%, \mathrm{p}=0.08)$. Six patients in the UFHtreated group developed HIT, 4 of whom had thrombotic events (HIT with thrombosis). In the LMWH-treated group, 3 patients developed HIT, 1 of whom had HIT with thrombosis. In the study by Fanikos et al. [12] compared efficacy, safety, and cost of low-molecular-weight heparin with continuous-infusion unfractionated heparin for initiation of anticoagulation after mechanical prosthetic valve implantation. Patients who received low-molecular-weight heparin had a shorter length of stay and decreased postoperative costs compared with the control subjects receiving unfractionated heparin. Significantly more bleeding complications in the warfarin plus heparin group versus warfarin group as evidenced by higher rates of pericardial effusions $(24 \%$ vs. $8 \%, \mathrm{p}=0.02)$ and reoperation for bleeding $(8 \% v$ s. $0 \%, \mathrm{p}=$ $0.05)$ was reported in retrospective study evaluating differences in bleeding and thrombotic events between a homogenous group of patients undergoing mechanical aortic valve replacement who either received or did not receive intravenous unfractionated heparin or subcutaneous low-molecular weight heparin as bridging strategy to warfarin therapy [13] . Logistic regression analysis identified group assignment (warfarin plus heparin versus warfarin only) to be significantly associated with the odds of bleeding (odds ratio 4.46, 95\% confidence interval:1.42 to $14.02, \mathrm{p}=0.01)$. A recent meta-analysis including twenty-three studies (9534 patients) reported bleeding rates of $1.8 \%$ (95\% confidence interval CI 1.0-3.3) in the group receiving OAC, $2.2 \%$ (95\% CI 0.9-5.3) in the OAC + UFH group, and $5.5 \%$ (95\% CI 2.9-10.4) in the OAC + LMWH group $(\mathrm{P}=0.042)$ [14]. The thromboembolic event rate was $2.1 \%$ (95\% CI 1.5-2.9) in the group receiving OAC, as compared with $1.1 \%$ (95\% CI 0.7-1.8) of OAC + UFH and $\mathrm{OAC}+\mathrm{LMWH}(\mathrm{P}=0.035)$.

\section{Limitations of the study}

The small number of patients included in present study hampers conclusive statements about the efficacy and safety of low molecular weight heparin as bridging therapy to oral anticoagulants after heart surgery. However present results confirm the observations of most of previous investigations as reported in the discussion section. Due to the protocol of randomization, for chance, the number of patients included in the enoxaparin group was about one third of overall population under investigation, nevertheless the clinical characteristics of the two groups did not differ significantly. At variance with previous studies in present investigation we included patients undergoing different surgical procedures, all needing long - term oral anticoagulation, at different risk of bleeding and thromboembolic complications in order to have more adherence to real world conditions.

\section{Conclusion}

Results from present pilot study suggest that patients who need prolonged oral anticoagulation in hospital bridging treatment with weight adjusted enoxaparin seems as safe as weight adjusted s.c unfractionated heparin with a lower rate of blood transfusion and a lower rate of pericardial effusion and need for pericardial drainage. Larger trials are necessary to establish the clinical relevance of bridging therapy and the safety of LMWH.

\section{Conflict of interest}

None declared

\section{References}

1. Butchart EG (2009) Antithrombotic management in patients with prosthetic valves: a comparison of American and European guidelines. Heart 95: 430-436. [Crossref]

2. Whitlock RP, Sun JC, Fremes SE, Rubens FD, Teoh KH (2012) Antithrombotic and thrombolytic therapy for valvular disease: Antithrombotic Therapy and Prevention of Thrombosis, 9th ed: American College of Chest Physicians Evidence-Based Clinical Practice Guidelines. Chest 141: e576S-e600S. [Crossref] 
Rostagno C (2017) Bridging therapy to chronic anticoagulant treatment in patients undergoing cardiac surgery: comparison between low-molecular-weight heparin and subcutaneous unfractionated heparin. A pilot study

3. Montalescot G, Polle V, Collet JP, Leprince P, Bellanger A, et al. (2000) Low molecular weight heparin after mechanical heart valve replacement. Circulation 101: 1083-1086. [Crossref]

4. Rivas-Gándara N, Ferreira-González I, Tornos P, Torrents A, Permanyer-Miralda G, et al. (2008) Enoxaparin as bridging anticoagulant treatment in cardiac surgery. Heart 94 205-210. [Crossref]

5. Meurin P, Tabet JY, Weber H, Renaud N, Ben Driss A (2006) Low-molecular-weight heparin as a bridging anticoagulant early after mechanical heart valve replacement. Circulation 113: 564-569. [Crossref]

6. Bucci C, Geerts WH, Sinclair A, Fremes SE (2011) Comparison of the effectiveness and safety of low-molecular weight heparin versus unfractionated heparin anticoagulation after heart valve surgery. Am J Cardiol 107: 591-594. [Crossref]

7. Stein PD, Alpert JS, Bussey HI, Dalen JE, Turpie AG (2001) Antithrombotic therapy in patients with mechanical and biological prosthetic heart valves. Chest 119: 220S-227S. [Crossref]

8. Schlitt A, von Bardeleben RS, Cannegieter SC, Rosendaal FR, Wintzen AR, et al. (1995) Optimal oral anticoagulant therapy in patients with mechanical heart valves. $N$ Engl J Med 333: 11-17. [Crossref]
9. Sun JC, Davidson MJ, Lamy A, Eikelboom JW (2009) Antithrombotic management of patients with prosthetic heart valves: current evidence and future trends. Lancet 374 : 565-576. [Crossref]

10. Guidelines on the management of valvular heartdisease (version 2012) (2012) The Joint Task Force on the Management of Valvular Heart Disease of the European Society of Cardiology (ESC) and the EuropeanAssociation for Cardio-Thoracic Surgery (EACTS) European Heart Journal 33: 2451-2496. [Crossref]

11. Nishimura RA, Otto CM, Bonow RO, Carabello BA, Erwin JP (2014) 2014 AHA/ACC guideline for the management of patients with valvular heart disease: a report of the American College of Cardiology/American Heart Association Task Force on Practice Guidelines. J Am Coll Cardiol 63: e57-185 [Crossref]

12. Fanikos J, Tsilimingras K, Kucher N, Rosen AB, Hieblinger MD, et al. (2004) Comparison of efficacy, safety, and cost of low-molecular-weight heparin with continuous-infusion unfractionated heparin for initiation of anticoagulation after mechanical prosthetic valve implantation. Am. J. Cardiol. 93: 247-250. [Crossref]

13. Guglielmetti LC, Sorabella R, Chiuzan C, Najjar M, Castillero E, et al. (2016) Bridging Anticoagulation After Mechanical Aortic Heart Valve Replacement: A Questionable Routine. Ann Thorac Surg 102: 48-54. [Crossref]

14. Passaglia LG, de Barros GM, de Sousa MR (2015) Early postoperative bridging anticoagulation after mechanical heart valve replacement: a systematic review and meta-analysis. J Thromb Haemost 13: 1557-67. [Crossref]

Copyright: $\odot 2017$ Rostagno C. This is an open-access article distributed under the terms of the Creative Commons Attribution License, which permits unrestricted use, distribution, and reproduction in any medium, provided the original author and source are credited. 